\title{
Dual-frequency cesium spin maser
}

\author{
P. Bevington $\odot,{ }^{1,2}$ R. Gartman, ${ }^{1}$ Y. V. Stadnik $\odot,{ }^{3}$ and W. Chalupczak $\oplus^{1}$ \\ ${ }^{1}$ National Physical Laboratory, Hampton Road, Teddington TW11 OLW, United Kingdom \\ ${ }^{2}$ Department of Physics, University of Strathclyde, Glasgow G4 ONG, United Kingdom \\ ${ }^{3}$ Kavli Institute for the Physics and Mathematics of the Universe (WPI), The University of Tokyo Institutes for Advanced Study, \\ The University of Tokyo, Kashiwa, Chiba 277-8583, Japan
}

(Received 20 January 2020; accepted 11 August 2020; published 2 September 2020)

\begin{abstract}
Comagnetometers have been validated as valuable components of the atomic physics toolbox in fundamental and applied physics. So far, the explorations have been focused on systems involving nuclear spins. Presented here is a demonstration of an active alkali-metal (electronic) system, i.e., a dual-frequency spin maser operating with the collective cesium $F=3$ and $F=4$ spins. The experiments have been conducted in both magnetically shielded and unshielded environments. In addition to the discussion of the system's positive-feedback mechanism, the implementation of the dual-frequency spin maser for industrial nondestructive testing is shown. The stability of the $F=3$ and $F=4$ spin precession frequency ratio measurement is limited at the $3 \times 10^{-8}$ level by the laser frequency drift, corresponding to a frequency stability of $1.2 \mathrm{mHz}$ for $10^{4} \mathrm{~s}$ integration time. We discuss measurement strategies that could improve this stability to $\mathrm{nHz}$, enabling measurements with sensitivities to the axion-nucleon and axion-electron interactions at the levels of $f_{a} / C_{N} \sim 10^{9}$ and $f_{a} / C_{e} \sim 10^{8} \mathrm{GeV}$, respectively.
\end{abstract}

DOI: 10.1103/PhysRevA.102.032804

\section{INTRODUCTION}

The idea of an alkali-metal spin maser system was introduced in the 1960s [1,2]. When exploring self-oscillating radio-frequency (rf) atomic systems, Bloom pointed out that "the response of the spin system with regard to the feedback loop is similar to that of a spin system in a maser (in which case the feedback loop consists of the radiation reaction inside the cavity)" [1]. As in other maser (or laser) systems, the alkali-metal spin maser includes population inversion in the form of spin polarization along a static bias magnetic field (i.e., population imbalance) within the ground-state manifold. Spontaneous fluctuations create spin components orthogonal to the bias field (i.e., atomic coherences) that precess around the magnetic field at the Larmor frequency. The collective atomic spin precession is optically monitored with a photodetector, the output of which is fed into rf coils located in the vicinity of the atomic vapor. The rf field that is generated provides positive feedback to the spontaneous spin precession, provided that the sum of all phase shifts in the feedback loop is zero. Initial studies of this system were limited to the area of geomagnetic measurements [3-6]. Extensive studies of spin maser properties have been performed in noble gases, where the term spin (or Zeeman) maser was coined and the idea of a dual-frequency spin maser (DFSM) was developed [7-11].

The concept of a DFSM, i.e., an active system with two spins simultaneously precessing in the same bias field, enables relative measurements and addresses the issue of drifts in the operating (Larmor) frequency. Consequently, this expands the area of possible applications beyond basic magnetic field measurements. DFSMs and comagnetometers (in this context, passive systems without a feedback loop) are particularly attractive for inertial sensing [12] and testing of fundamental physics [13-15]. In noble gases, DFSMs have been achieved in mixtures of ${ }^{3} \mathrm{He}$ and ${ }^{129} \mathrm{Xe}$ [9-11] or ${ }^{129} \mathrm{Xe}$ and ${ }^{131} \mathrm{Xe}$ [14]. While operation at two frequencies makes the active and passive systems insensitive to drifts in the bias magnetic field, there remains a whole spectrum of fundamental and technical shifts that compromise the frequency ratio measurement. From this perspective, a particularly interesting demonstration was the operation of a comagnetometer within the same molecule [16]. The operation of a comagnetometer or spin maser within identical molecules or atoms, rather than overlapping ensembles of different atoms, reduces the noise contribution due to magnetic field gradients.

In this paper, we demonstrate an alkali-metal DFSM within the same isotope of a single element. We discuss in detail the principle of operation, i.e., the mechanism for positive feedback, the basic properties of the system, and explore possible applications. We investigate a spin maser simultaneously operating with the $F=3$ and $F=4$ ground-state hyperfine level components of the collective spin of an ensemble of cesium atoms. Adjustment of the beam power (pump and probe) and the probe laser frequency detuning from the relevant transitions enables the creation of a signal with equal amplitudes and phases and, consequently, provides positive feedback for the $F=3$ and $F=4$ spins, even though they precess in opposite directions.

The experiments were performed in both magnetically unshielded and shielded environments, demonstrating the system capabilities in both industrial [nondestructive testing (NDT)] and fundamental studies, respectively. The former is based on magnetic inductive measurements with an electrically conductive or magnetically permeable object. The object's response to an oscillating rf magnetic, so-called primary, field indicates the presence of structural defects and 
composition inhomogeneities within the studied sample. Implementation of the spin maser concept helps to overcome some of the important technological challenges in NDT with rf atomic magnetometers, in particular the stability of the bias field in the presence of a ferromagnetic object and the image acquisition time [17-20]. The latter is demonstrated through the Larmor frequency ratio measurement between the two hyperfine ground-state levels, which has applications in the measurement of the $g_{i} / g_{j}$ ratio [21] or for the possible detection of dark-matter candidates, i.e., axion wind, through interaction with the atomic spins $[15,16,22]$. The dark-matter searches are the focus of many theoretical and experimental efforts, and ultrasensitive atomic magnetometers are one of the systems being used in this pursuit $[23,24]$. The DFSM demonstrated in this work presents another tool for dark-matter searches over a particular parameter space. The realization of the experiment described here may not be best suited for the frequency ratio measurement, so we propose some practical changes that could improve the stability by several orders of magnitude.

\section{EXPERIMENTAL SETUP}

The measurements described here are performed in two configurations, i.e., shielded [25,26] and unshielded [17-20], both of which have been described before and here only their essential elements are recalled. In both setups, cesium atomic vapor is housed in a paraffin-coated cell at ambient temperature (atomic density $n_{\mathrm{Cs}}=0.33 \times 10^{11} \mathrm{~cm}^{-3}$ ), where we take advantage of the laboratory's temperature stability $\left( \pm 0.2^{\circ} \mathrm{C}\right)$. Pumping is performed by a circularly polarized laser beam, frequency stabilized to the $6^{2} S_{1 / 2} F=3 \rightarrow$ $6^{2} P_{3 / 2} F^{\prime}=2$ transition $\left(D_{2}\right.$ line, $\left.852 \mathrm{~nm}\right)$ [see Fig. 1(a)], propagating along the direction of the bias static magnetic field see Fig. 1(b). For the purpose of the frequency ratio measurement, the pump beam power is stabilized with a proportional-integral-differential controller that modifies the rf input to an acousto-optic modulator installed in the pump beam's optical path. Paramagnetic Faraday rotation maps the instantaneous value of the collective atomic spin onto the polarization state of the linearly polarized probe beam [26-28] that propagates orthogonally to the pump beam. The probe laser frequency is $2.75 \mathrm{GHz}$ red detuned from the $6^{2} S_{1 / 2}$ $F=3 \rightarrow 6^{2} P_{3 / 2} F^{\prime}=2$ transition via phase offset locking to the pump beam. The resulting signal is either measured by a lock-in amplifier referenced to the first harmonic of the rf field frequency or recorded by a $2 \mathrm{MS} / \mathrm{s}$ data-acquisition board. For the DFSM, the optical polarization-rotation signal is a beatnote of the two $F=3$ and $F=4$ frequency components. This beatnote signal is fed into a rf coil in the vicinity of the cell to provide the driving feedback rf field to the atoms.

In the shielded setup, the ambient magnetic field is suppressed by the use of five layers of cylindrical shields with end caps, made from 2-mm-thick mu-metal. A pair of solenoids inside the shield generates a well-controlled bias magnetic field with a relative homogeneity exceeding $10^{-4}$ over the length of the cell. A set of Helmholtz coils around the vapor cell creates the driving rf field.

In the unshielded setup, the bias field is defined by a set of nested, orthogonal, square Helmholtz coils. The square

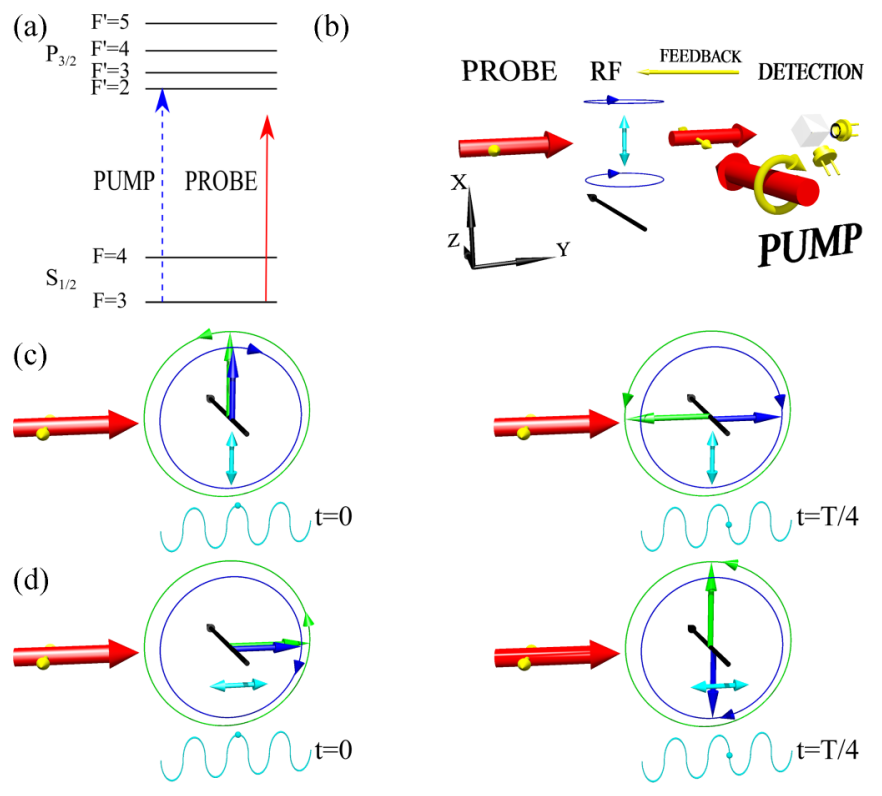

FIG. 1. (a) Cesium $D_{2}$ line ( $852 \mathrm{~nm}$ ) energy structure (pump and probe laser frequencies marked with dashed blue and solid red lines). (b) Spin maser operating simultaneously at the $F=3$ and $F=4$ Larmor frequencies. The $F=3$ and $F=4$ atomic spins are oriented by the pump beam along the bias magnetic field (black arrow) parallel to the $\hat{z}$ axis. The spin evolution is monitored continuously by the linearly polarized probe beam propagating along the $\hat{y}$ axis. (c),(d) Evolution of the atomic spins in two configurations of the continuous-wave rf field. The direction of the rf field is marked by the cyan arrow, while the relevant time point in the temporal field amplitude oscillation is illustrated by a dot on the cyan curve. Green and blue arrows represent components of the $F=3$ and $F=4$ spins (coherences) precessing in the bias magnetic field. At $t=0$, these spin components are created in the same direction as the rf field. The two components then proceed to precess in opposite directions. When there is a nonzero projection of the spins on the probe beam (red arrow) axis, a nonzero polarization-rotation signal is observed. The $F=3$ and $F=4$ spin components after a quarter of an evolution period are shown in the picture labeled $t=T / 4$.

Helmholtz coils have lengths of $2 a=1,0.94$, and $0.88 \mathrm{~m}$ (separation of $0.5445 a$ ), respectively, such that they generate a homogeneous field (at the $10^{-3}$ level) over the volume of the vapor cell. The rf primary field is generated by a single coil (300 turns of $0.2 \mathrm{~mm}$ diameter copper, wound length of $3 \mathrm{~mm}$, inner and outer diameters of 2 and $4 \mathrm{~mm}$ ) with a ferrite core (length $6 \mathrm{~mm}$ and diameter $2 \mathrm{~mm}$ ). The coil is placed 2-7 mm from the object on the same axis as the cell and $200 \mathrm{~mm}$ from the atomic magnetometer.

\section{DUAL-FREQUENCY SPIN MASER}

\section{A. The $F=3$ and $F=4 \mathrm{rf}$ spectra}

The pump beam is directly coupled to the $F=3$ level, making the precession of the $F=3$ spin component prone to perturbations caused by instabilities in the laser amplitude and frequency. Implementation of low power pumping (up to $2 \mu \mathrm{W}$ ) and laser power stabilization partially addresses this problem. The pumping of the $F=4$ collective spin is achieved by indirect pumping [25], i.e., a combination of 

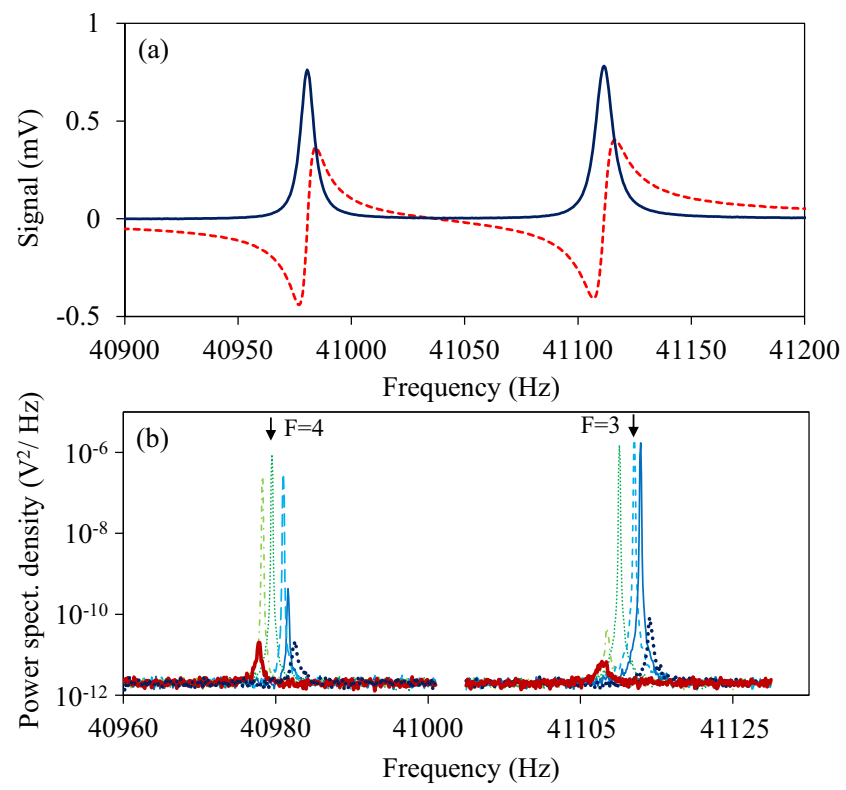

FIG. 2. (a) The spectrum of the $\mathrm{rf}$ transition within the $F=4$ and $F=3$ ground-state levels (marked with black arrows) for a bias magnetic field of $4.5 \mu \mathrm{T}$. Solid (blue) and dashed (red) lines represent the in-phase and quadrature components recorded by the lock-in amplifier. (b) Power spectral density of the DFSM signal showing a change of the operating frequency for six values of the phase shift in the feedback loop. The solid thick red and the thick dotted dark-blue line profiles represent amplified spontaneous fluctuations. The data in both (a) and (b) are recorded in the shielded setup.

off-resonant excitation and spin-exchange collisions, resulting in a decoupling of the $F=4$ spin precession frequency from the laser light in the power range used in these measurements. Radio-frequency spectroscopy is used to monitor the spin polarization within the $F=3$ and $F=4$ ground-state hyperfine levels [25]. Figure 2(a) shows a typical polarizationrotation signal measured as a function of the rf field frequency recorded in the shielded setup. Resonances are observed when the rf field frequency matches the splitting between neighboring Zeeman sublevels introduced by the bias field. The difference in Landé factors for the $F=3$ and $F=4$ groundstate hyperfine levels results in opposite signs of Larmor frequency and a separation in the respective resonance frequencies. In Fig. 2(a), the solid (blue) and dashed (red) lines represent the two components of the signal recorded by a lockin amplifier. As the frequency of the rf driving field is scanned across the rf resonance, the phase of the atomic response changes by $180^{\circ}$. Most of the phase change occurs within the frequency span between the minimum and maximum of the dispersivelike component (red dashed line).

\section{B. Dual-frequency spin maser geometry}

Operation of DFSM requires the presence of positive feedback, i.e., the rf field driven by the signal from the balanced photodetector must be in phase with the precession of both the $F=3$ and $F=4$ atomic spins. To provide positive feedback for the case where the rf field axis is orthogonal to the probe beam propagation direction, the photodetector signal must be shifted by $90^{\circ}$. If they are parallel, no phase shift is necessary. The sign of the detected signal is a product of the amplitude of the atomic spin component along the probe beam direction and the sign of the detuning from the relevant atomic resonance. The positive-feedback condition for both spins amounts to selecting the right sign of the probe beam detuning from the $F=3$ and $F=4$ resonances for a specific direction of the rf field. In other words, the detuning ensures that optical rotation of the probe due to the $F=3$ and $F=4$ components occurs in the same direction. An intuitive explanation of the DFSM operation in terms of the $F=3$ and $F=4$ spin components (coherences) precessing in the bias magnetic field is provided in Fig. 1. We first consider the configuration where the rf field [cyan arrow in Figs. 1(b)1(d)] direction is orthogonal to the probe beam propagation direction [Fig. 1(c)]. The $F=3$ and $F=4$ atomic spins are oriented by the pump beam along the bias magnetic field axis, and subsequently tilted by the rf field in the same direction (see pictures labeled $t=0$ in Fig. 1). The two components then proceed to precess in opposite directions, and hence the projections of either spin component on the probe beam axis have opposite signs (see pictures labeled $t=T / 4$ in Fig. 1). If the probe beam frequency is set between the $F=3$ and $F=4$ resonances, the two components have an opposite detuning. Hence, the signals for the $F=3$ and $F=4$ components (amplitude of the demodulated output of the lock-in amplifier) have the same sign. For the configuration where the rf field direction is parallel to the probe beam propagation direction [Fig. 1(d)], the DFSM operation requires the same sign of detuning for both the $F=3$ and $F=4$ atoms. Thus, the probe beam frequency has to be tuned above or below the $F=3$ or $F=4$ group of transitions, respectively. Through the analysis of the spin maser action in an NDT measurement, the following results explicitly describe how the operation of a DFSM depends on various rf field geometries and probe beam detunings.

\section{Nondestructive testing with a dual-frequency spin maser}

In inductive measurements, the atomic spins are exposed to the primary field created by the excitation coil and the secondary field, i.e., the response of the studied object to the primary field. The measurement geometry used in the experiments described here (direction of bias field and primary field both parallel to the surface normal of the object) means that the atomic spin precession is only driven by the secondary fields perpendicular to the surface normal of the object [19]. In other words, the feedback that supports the maser action is solely created by the components of the secondary field [20]. The amplitude of these components is only nonzero when the rf excitation coil is in the vicinity of a defect (recess).

The condition for positive feedback in the spin maser has two requirements: (1) the gain in the feedback loop exceeds the losses in the system and (2) the phase matching between the spontaneous spin precession and the secondary field. The phase-matching condition means that the sum of all phase shifts (i.e., secondary field, atomic signal, and external phase shift) in the feedback loop is zero [1].

The angular distribution of the secondary field produced by a circular recess in our NDT measurement configuration 
(a)

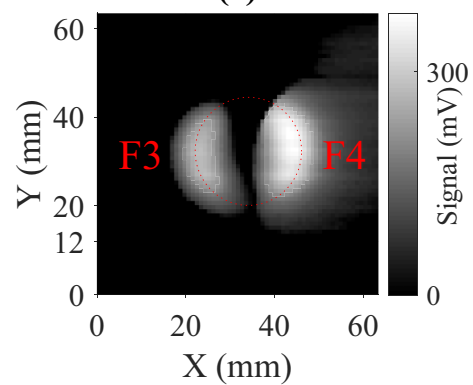

(d)

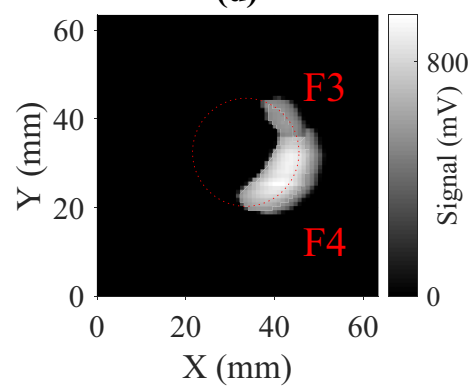

(b)

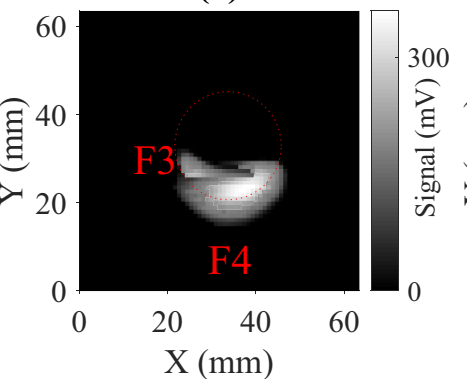

(e)

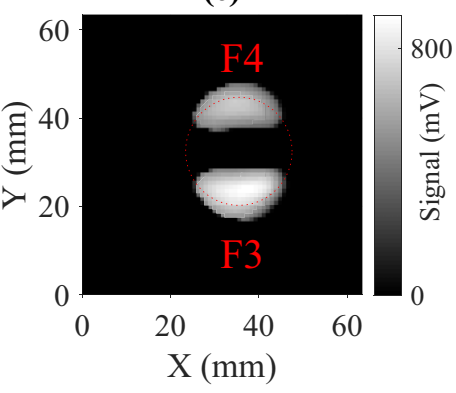

(c)

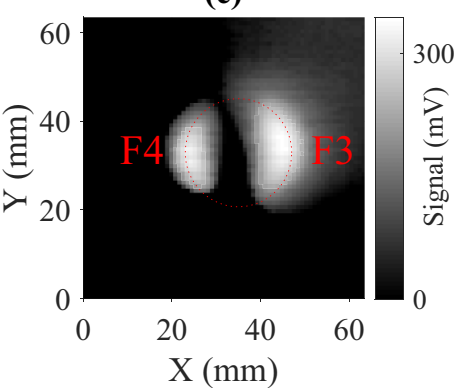

(f)

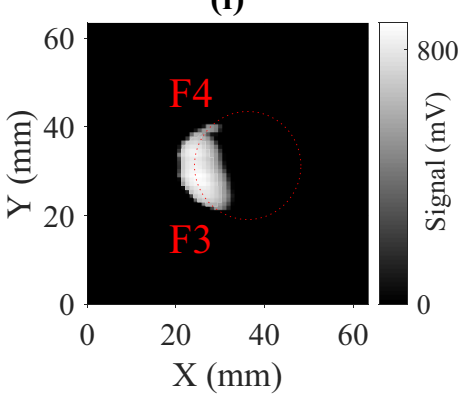

FIG. 3. Change in the dual-frequency spin maser signal amplitude recorded over a $64 \times 64 \mathrm{~mm}^{2}$ area of a 6-mm-thick aluminium plate containing a 24-mm-diameter recess that is $2.4 \mathrm{~mm}$ deep (outlined with dashed red circle) for (a),(d) $0^{\circ}$, (b), (e) $90^{\circ}$, and (c),(f) $180^{\circ}$ phase shifts in the feedback loop. Images (a)-(c) were recorded with the probe $2.75 \mathrm{GHz}$ red detuned from the $6^{2} S_{1 / 2} F=3 \rightarrow 6^{2} P_{3 / 2} F^{\prime}=2$ transition. Images (d)-(f) were recorded with the probe beam frequency $890 \mathrm{MHz}$ blue detuned from the $6^{2} S_{1 / 2} F=3 \rightarrow 6^{2} P_{3 / 2} F^{\prime}=2$ transition. Measurements were performed with a Larmor frequency $\sim 40 \mathrm{KHz}$.

changes continuously from $0^{\circ}-360^{\circ}$ around the perimeter of the recess. This continuous change in direction of the secondary field [Figs. 1(c) and 1(d) show field orientations, with an angle of $90^{\circ}$ between them] is equivalent to a continuous change of the phase of the rf field that drives the feedback loop, ensuring that the phase-matching condition will always be met over some section of the defect. However, this is a downside of spin maser operation when compared to the freerunning mode (i.e., with an external drive for the rf field) [19] since it does not record the full defect signature [20]. Application of the DFSM helps to recover a larger section of the defect signature.

The images in Fig. 3 show the change of the DFSM amplitude recorded over a $64 \times 64 \mathrm{~mm}^{2}$ area of a 6 -mm-thick $\mathrm{Al}$ plate containing a $24-\mathrm{mm}$-diameter recess that is $2.4 \mathrm{~mm}$ deep, which mimics local thinning due to structural anomalies such as corrosion or fatigue [17]. The columns in the image array represent three different external phase shifts in the feedback loop $\left(0^{\circ}, 90^{\circ}\right.$, and $\left.180^{\circ}\right)$ and the two rows reflect two different probe beam detunings $(-2.75 \mathrm{GHz}$ and $890 \mathrm{MHz})$ from the $6^{2} S_{1 / 2} F=3 \rightarrow 6^{2} P_{3 / 2} F^{\prime}=2$ transition. The different phase shifts applied in the feedback loop are equivalent to the two configurations shown in Fig. 1. In particular, a $0^{\circ}$ phase shift represents the field geometry in Fig. 1(d), while $90^{\circ}$ phase shifts are equivalent to the field geometry in Fig. 1(c). A change in the phase shift and probe laser detuning are compensated for by the adjustment of the phase of the secondary field. This results in the $F=3$ and $F=4$ spin maser operation being triggered at different sections around the edge of the recess.

For a phase shift of $0^{\circ}$ [Figs. 3(a) and 3(d)], spin maser (SM) action occurs when the secondary field is directed along the probe beam [Fig. 1(d), $t=0$ ]. For Fig. 3(a), the detuning for the $F=3$ and $F=4$ spins is opposite and, consequently, so will be the sign of their signals, resulting in phase matching at opposite sides of the recess. The detuning in Fig. 3(d) is the same for $F=3$ and $F=4$, so phase matching is met over the same section of the recess. Figures 3(b) and 3(e) represent the case with a $90^{\circ}$ phase shift, i.e., the secondary field is orthogonal to the probe beam [Fig. 1(c), $t=0$ ]. The secondary field tilts the $F=3$ and $F=$ 4 spin in the same direction, but they precess clockwise and counterclockwise, respectively, resulting in opposite projections along the probe beam axis [Fig. 1(d), $t=T / 4$ ]. This time, for Fig. 3(b), the opposite sign of detuning leads to overlapping regions of SM action. For the detuning in Fig. 3(e), the phase-matching conditions are met at opposite sides of the recess. By symmetry, a phase shift of $180^{\circ}$ [Figs. 3(c) and 3(f)] reflects the opposite case to Figs. 3(a) and 3(d).

We note that in regions of overlapping $F=3$ and $F=4$ maser action, fast Fourier transform (FFT) measurements indicate that the system only oscillates at one of the two possible frequencies and shows behavior typical for a bistable system. This effect has also been observed in a shielded system where the feedback is produced directly by the rf coils. By recording the signals with a fast data-acquisition board, we confirmed that the effect is not simply due to the use of a lock-in amplifier (can only detect signals at a single frequency). We ascribe it to the coupling between two oscillating modes [29-31], and this aspect of the system behavior will be discussed elsewhere.

\section{Dual-frequency spin maser spectrum}

Figure 2(b) shows the power spectral density of the maser signal recorded for various values of the phase shift in the 
feedback loop recorded in the shielded setup. As with any driven oscillator, the phase of the atomic response varies by $180^{\circ}$ if the frequency of the driving $\mathrm{rf}$ field is tuned across resonance. The spin maser will operate at whichever frequency, within the rf resonance profile, leads to an overall phase shift of zero [5,6]. Consequently, as shown in Fig. 2(b), the operating frequency changes for different external phase shifts, the rate of which is set by the linewidth of the rf resonance; see Fig. 2(a). This is particularly important when considering a frequency ratio measurement, which is addressed in the following section. For large phase shifts $\left(\approx \pm 90^{\circ}\right)$, the response to the drive field is manifested as a broad, low-amplitude peak in the power density spectrum. This is the spin equivalent of amplified spontaneous emission in laser systems. The signal is created by spin fluctuations that are fed into the rf coil with positive feedback that has a strength comparable with the decoherence rate. The frequency spread of the signal is defined by the decoherence rate and the inhomogeneity of the bias magnetic field.

\section{E. Frequency ratio measurement}

Comagnetometers can be used in searches for anomalous spin-dependent interactions (which may arise, e.g., via the interaction of spins with dark matter) by searching for timedependent signals in measurements of the Larmor frequency ratio of two different species $[13,15,22,32]$, or various timeindependent signals $[33,34]$. We discuss this measurement, i.e., the measurement of the ratio between the $F=3$ and $F=4$ spin precession frequencies, in order to illustrate the capabilities and challenges of implementing a DFSM in fundamental physics explorations.

A continuous frequency ratio measurement was divided into 100-s-long sections. The resonance frequencies of the $F=3$ and $F=4$ components were extracted from the FFT spectrum of each section. Figure 4 shows the statistical uncertainty (Allan deviation) of the frequency of the $F=3$ and $F=4$ spin maser components (green bars and black crosses) as well as their ratio (red triangles). In order to show all the results in one plot, the relative uncertainty of each parameter has been calculated. The short-term stability of the relative uncertainty of the $F=3$ and $F=4$ frequency measurement is set at the $\approx 2 \times 10^{-6}$ level by the stability of the current source. For $2 \times 10^{2}<\tau<2 \times 10^{3} \mathrm{~s}$, the behavior of their statistical uncertainties is dominated by thermal drifts of the bias magnetic field. The frequency ratio (red triangles) is insensitive to variations in the magnetic field and it follows $\mathrm{a} \sim \tau^{-1 / 2}$ trend (red dashed line) for $\tau<10^{4} \mathrm{~s}$, indicating a dominant white-noise character. Evaluation of the stability limits involves the following: (1) a measurement of the slope of the frequency ratio dependence on various measurement parameters, such as laser beam power and laser frequency stability, and (2) monitoring the parameters' value over the course of the frequency ratio measurements. While the longterm stability of the $F=3$ to $F=4$ frequency ratio is not affected by the laser beams' powers (dependence on the pump beam power represented by black dots), the Allan deviation of the frequency ratio (red triangles) for $\tau>10^{4} \mathrm{~s}$ mirrors that of the limit set by the pump laser frequency noise (blue diamonds). The laser frequency drift limits the stability of the

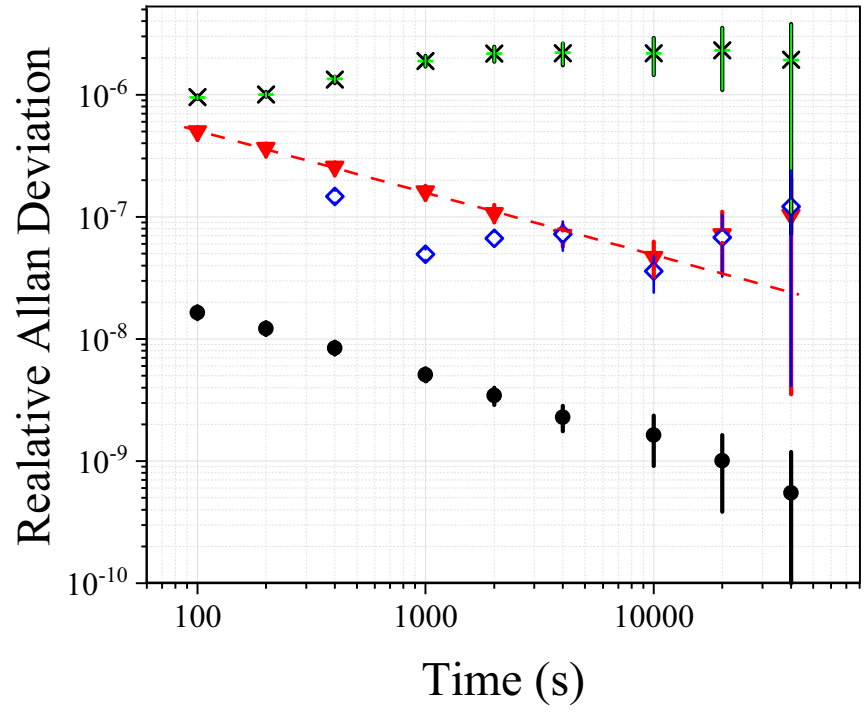

FIG. 4. The statistical uncertainty (Allan deviation) of the frequency of the $F=3$ and $F=4$ spin maser components (green bars and black crosses) recorded in the shielded setup. In order to show all the results in one plot, the relative uncertainty of each parameter has been calculated. The behavior of the Allan deviation indicates flicker noise, which results from the temperature-driven variations in the magnetic field. The initial dependence of the statistical uncertainty of the frequency ratio (red triangles) on integration time $\left(\sim \tau^{-\frac{1}{2}}\right)$ indicates a dominant "white-noise" character. The black points and blue diamonds represent the limits imposed by the two leading contributions: the pump beam power and frequency stability. The bias magnetic field is set to $11.4 \mu \mathrm{T}$, at which the Larmor frequency $\approx$ $40 \mathrm{KHz}$.

$F=3$ and $F=4$ spin precession frequency ratio measurement at the $3 \times 10^{-8}$ level, which corresponds to a frequency stability of $1.2 \mathrm{mHz}$ for $10^{4} \mathrm{~s}$ integration time. The drifts in the laser frequency stabilization are an artifact of the poor optimization of the temperature-stabilization unit in the laser diode controller [35]. We verified that fitting a waveform to the raw signal [with the signal-to-noise level $(\mathrm{SNR}) \sim 10^{2}$ ] can improve the accuracy of the frequency measurement by $10^{2}$, compared with the limit defined by the FFT resolution. Exploiting this allows us to reach an uncertainty in the frequency ratio of $\approx 10^{-10}$, equivalent to the frequency stability at the $\approx 4 \mu \mathrm{Hz}$ level after $10^{4} \mathrm{~s}$ integration time [14,15]. In the context of searches for axionlike dark matter via spin precession about Earth's direction of motion through the galactic dark matter [36], this would translate into sensitivities to the axion-nucleon and axion-electron interactions at the levels of $f_{a} / C_{N} \sim 10^{5}$ and $f_{a} / C_{e} \sim 10^{4} \mathrm{GeV}$, respectively, for the axion masses $m_{a} \lesssim 10^{-18} \mathrm{eV} / c^{2}$, which are particularly interesting from an astrophysical point of view [37]. The axion decay constant $f_{a}$ (which has dimensions of energy) and the model-dependent dimensionless parameters $C_{N, e}$ are defined via the Hamiltonian $H_{\text {int }} \sim\left(C_{\psi} \sqrt{\rho} / f_{a}\right) \sigma_{\psi} \cdot \mathbf{v}_{a} \sin \left(m_{a} t\right)$, where $\rho \approx 0.4 \mathrm{GeV} / \mathrm{cm}^{3}$ denotes the local dark-matter energy density, $\sigma_{\psi}$ denotes the spin of fermion $\psi=N, e$, and $\mathbf{v}_{a} \sim$ $10^{-3} c$ is the speed of the axion dark-matter flux relative to Earth. 
While further improvements of the setup are possible, the direct optical coupling to the $F=3$ state makes the presented system suboptimal for a frequency ratio measurement. As an alternative, the two naturally occurring isotopes of $\mathrm{Rb}$ could be explored. Specifically, implementation of the $F=3\left({ }^{85} \mathrm{Rb}\right)$ and $F=2\left({ }^{87} \mathrm{Rb}\right)$ spin ensemble in the alkali-metal DFSM could take full advantage of the indirect pumping process. Indirect pumping of both isotopes configured as in Ref. [25], i.e., off-resonant pumping with a laser operating at the $D_{2}$ transition combined with spin-exchange collisions, would ensure decoupling of the target states from the optical field. This would reduce the sensitivity of the frequency ratio measurement on the pump beam frequency and power stability. Additionally, as demonstrated in [25], optimized pump power can lead to the transfer of up to $82 \%$ of the atomic population in the target state. This results in an increase in the SNR of $\sim 10^{5}$ reported in [26], with respect to the case discussed here, where partial (no-optimized) orientation is essential for simultaneous observation of both spin components. Consequently, the discussed approach would enable measurements with a frequency stability at the $\mathrm{nHz}$ level and, therefore, at the levels of $f_{a} / C_{N} \sim 10^{9}$ and $f_{a} / C_{e} \sim 10^{8} \mathrm{GeV}$ for axion-nucleon and axion-electron interactions, respectively. This would improve the sensitivity to the axion-electron coupling strength with respect to spin-polarized torsion pendulum experiments [38] by about two orders of magnitude.

\section{CONCLUSION}

In conclusion, we have demonstrated the operation of a cesium DFSM in magnetically shielded and unshielded environments, with the identification of the conditions required for positive feedback for various measurement configurations. From the perspective of fundamental research, the alkali-metal DFSM is a rich system that provides a platform for the exploration of self-oscillating systems and as a potential tool for dark-matter searches. In the context of industrial applications, self-oscillation systems enable operation in noisy (unstable) magnetic environments with a bandwidth which is not limited by the long ground-state coherence time [39].

\section{ACKNOWLEDGMENTS}

The work was funded by the UK Department for Business, Innovation and Skills as part of the National Measurement System Program. P.B. was supported by the Engineering and Physical Sciences Research Council (EPSRC) (Grant No. EP/P51066X/1). The work of Y.V.S. was supported by the World Premier International Research Center Initiative (WPI), MEXT, Japan, and by the JSPS KAKENHI Grant No. 20K14460.
[1] A. L. Bloom, Appl. Opt. 1, 61 (1962).

[2] W. E. Bell and A. L. Bloom, U.S. Patent No. 3257608A (1966).

[3] P. Dyal, R. T. Johnson, and J. C. Giles, Rev. Sci. Instrum. 40, 601 (1969).

[4] T. Kubo, Appl. Opt. 11, 1521 (1972).

[5] T. Kubo, M. Kondo, M. Kikuchi, K. Ohkawa, and S. Ando, J. Phys. E: Sci. Instrum. 6, 362 (1973).

[6] T. Yabuzaki and T. Ogawa, J. Appl. Phys. 45, 1342 (1974).

[7] R. E. Slocum, P. Clayton Cabiness, Jr., and S. Blevins, Rev. Sci. Instrum. 42, 763 (1971).

[8] M. G. Richards, B. P. Cowan, M. F. Secca, and K. Machin, J. Phys. B: At. Mol. Opt. Phys. 21, 665 (1988).

[9] T. E. Chupp, R. J. Hoare, R. L. Walsworth, and B. Wu, Phys. Rev. Lett. 72, 2363 (1994).

[10] D. Bear, T. E. Chupp, K. Cooper, S. DeDeo, M. Rosenberry, R. E. Stoner, and R. L. Walsworth, Phys. Rev. A 57, 5006 (1998).

[11] R. E. Stoner, M. A. Rosenberry, J. T. Wright, T. E. Chupp, E. R. Oteiza, and R. L. Walsworth, Phys. Rev. Lett. 77, 3971 (1996).

[12] J. Kitching, S. Knappe, and E. A. Donley, IEEE Sensors J. 11, 1749 (2011).

[13] C. Abel, N. J. Ayres, G. Ban, G. Bison, K. Bodek, V. Bondar, M. Daum, M. Fairbairn, V. V. Flambaum, P. Geltenbort et al., Phys. Rev. X 7, 041034 (2017).

[14] T. Sato, Y. Ichikawa, S. Kojima, C. Funayama, S. Tanaka, T. Inoue et al., Phys. Lett. A 382, 588 (2018).

[15] T. Wu, J. W. Blanchard, G. P. Centers, N. L. Figueroa, A. Garcon, P. W. Graham et al., Phys. Rev. Lett. 122, 191302 (2019).
[16] T. Wu, J. W. Blanchard, D. F. Jackson Kimball, M. Jiang, and D. Budker, Phys. Rev. Lett. 121, 023202 (2018).

[17] P. Bevington, R. Gartman, W. Chalupczak, C. Deans, L. Marmugi, and F. Renzoni, Appl. Phys. Lett. 113, 063503 (2018).

[18] P. Bevington, R. Gartman, and W. Chalupczak, Rev. Sci. Instrum. 90, 013103 (2019).

[19] P. Bevington, R. Gartman, and W. Chalupczak, J. Appl. Phys. 125, 094503 (2019).

[20] P. Bevington, R. Gartman, and W. Chalupczak, Appl. Phys. Lett. 115, 173502 (2019).

[21] J. Mora, A. Cobos, D. Fuentes, and D. F. J. Kimball, Ann. Phys. 531, 1800281 (2018).

[22] Y. V. Stadnik, Manifestations of Dark Matter and Variations of the Fundamental Constants of Nature in Atoms and Astrophysical Phenomena (Springer, Cham, Switzerland, 2017).

[23] M. Pospelov, S. Pustelny, M. P. Ledbetter, D. F. Jackson Kimball, W. Gawlik, and D. Budker, Phys. Rev. Lett. 110, 021803 (2013).

[24] S. Afach, D. Budker, G. DeCamp, V. Dumont, Z. D. Grujić, H. Guo et al., Phys. Dark Universe 22, 162 (2018).

[25] W. Chalupczak, R. M. Godun, P. Anielski, A. Wojciechowski, S. Pustelny, and W. Gawlik, Phys. Rev. A 85, 043402 (2012).

[26] W. Chalupczak, R. M. Godun, S. Pustelny, and W. Gawlik, Appl. Phys. Lett. 100, 242401 (2012).

[27] Y. Takahashi, K. Honda, N. Tanaka, K. Toyoda, K. Ishikawa, and T. Yabuzaki, Phys. Rev. A 60, 4974 (1999).

[28] I. M. Savukov, S. J. Seltzer, M. V. Romalis, and K. L. Sauer, Phys. Rev. Lett. 95, 063004 (2005). 
[29] G. P. Agrawal, and C. Flytzanis, IEEE J. Quantum Electron., 17, 374 (1981).

[30] G. Raithel, O. Benson, and H. Walther, Phys. Rev. Lett. 75, 3446 (1995).

[31] Y. P. Emelianova, V. V. Emelyanov, and N. M. Ryskin, Commun. Nonlinear Sci. Numer. Simul. 19, 3778 (2014).

[32] I. M. Bloch, Y. Hochberg, E. Kuflik, and T. Volansky, J. High Energy Phys. 01 (2020) 167.

[33] G. Vasilakis, J. M. Brown, T. W. Kornack, and M. V. Romalis, Phys. Rev. Lett. 103, 261801 (2009).

[34] P. Fadeev, Y. V. Stadnik, F. Ficek, M. G. Kozlov, V. V. Flambaum, and D. Budker, Phys. Rev. A 99, 022113 (2019).
[35] Temperature oscillation and drift were observed in early models of the Vescent laser and require replacement of the temperaturestabilization unit.

[36] Y. V. Stadnik and V. V. Flambaum, Phys. Rev. D 89, 043522 (2014).

[37] H.-Y. Schive, T. Chiueh, and T. Broadhurst, Nat. Phys. 10, 496 (2014).

[38] W. A. Terrano, E. G. Adelberger, C. A. Hagedorn, and B. R. Heckel, Phys. Rev. Lett. 122, 231301 (2019).

[39] P. D. D. Schwindt, L. Holberg, and J. Kitching, Rev. Sci. Instrum. 76, 126103 (2005). 